\title{
EL PERIODO JUVENIL VISTO DESDE LA PERSPECTIVA ADULTA
}

\author{
Rolando Pérez* \\ Wendy Aguilar** \\ David Víquez ${ }^{* * *}$
}

\section{RESUMEN}

Se indagó en las representaciones sobre la juventud desde la perspectiva de los adultos de dos situaciones sociales e institucionales diferentes. Los resultados muestran que ambos grupos ponen de relieve las diferencias entre la juventud de su propia generación y la juventud actual. Dichas diferencias refieren tanto a cambios tecnológicos, socio-culturales, económicos como a diferencias en el sistema de valores y prácticas de crianza que caracteriza una y otra generación. Los docentes realizaron una evaluación claramente más negativa sobre la generación actual, observando una pérdida de la autoridad adulta. Mientras que los padres identifican una corresponsabilidad en el comportamiento juvenil actual.

PALABRAS CLAVE: REPRESENTACIONES * JUVENTUD * MEDIOS DE COMUNICACIÓN * CATEGORIZACIÓN SOCIAL * ESQUEMAS DE GRUPOS * IDENTIDAD SOCIAL * RELACIONES INTERGENERACIONALES

\section{ABSTRACT}

The present study seeks to inquire the representations of youth from the adult perspective in two different social situations. The results show that both groups manifest the differences between the youth of its own generation and the present youth. These differences refer to economic, socio-cultural and technological changes, as well to differences in the value system and parenting practices that characterize one and the other generations. The teachers clearly evaluated in a negative way the present generation more than the parents did. The teachers observe a loss in the adult authority above the youth, while parents identify a co-responsibility in the present youth behavior.

KEYWORDS: REPRESENTATIONS $*$ YOUTH $*$ MASS MEDIA $*$ SOCIAL CATEGORIZATION * GROUPS SCHEMATA * SOCIAL IDENTITY * INTERGENERATIONAL RELATIONS.

\footnotetext{
* Instituto de Investigaciones Psicológicas, Universidad de Costa Rica (UCR).

rolarez@gmail.com

** Instituto de Investigaciones Psicológicas, Universidad de Costa Rica (UCR) y de la Fundación Omar Dengo. wvarlessa@gmail.com
}

*** Instituto de Investigaciones Psicológicas (UCR). davidviquez@gmail.com 


\section{INTRODUCCIÓN}

La construcción social de la relaciones intergeneracionales que ha aconteciendo en las sociedades occidentales en los últimos 60 años ha venido a dinamizar las relaciones intrafamiliares, ha contribuido a la diferenciación de los grupos juveniles, la organización del mundo laboral y a la transformación de la transmisión cultural. Esta construcción social tiene como núcleo la organización de las relaciones en torno a representaciones socialmente compartidas sobre los grupos generacionales, en este caso sobre la juventud y la adultez. Sobre la base de estas representaciones es que se definen expectativas recíprocas de acción que conforman el contenido de las relaciones intergeneracionales.

El tema de la juventud, en concreto, está presente de forma constante en la agenda pública. Deserción escolar, deficiencias al nivel educativo, criminalidad, drogas, falta de oportunidades de educación y empleo, pobreza, "apatía", alienación, "falta de límites", "desinterés" político, social o educativo, son algunos temas que cotidianamente se tematizan en los medios de comunicación, son retomados por los partidos políticos, por padres y madres o están presentes en las reflexiones de los educadores. Esto ha llevado muchas veces a la aparición del llamado pánico moral, demonizando a la juventud o a sectores de este, convirtiéndolos en elementos amenazantes o desintegradores de la sociedad. La aparición de este fenómeno de pánico moral lleva por lo general a una sobregeneralización y a una homogenización de la juventud o de algunas de sus expresiones grupales.

El abordaje teórico de la juventud que guía este texto supone la imbricación de dos perspectivas. La primera considera la juventud como un período del curso de la vida. En los últimos cincuenta años las sociedades altamente modernizadas o en proceso de modernización han experimentado una serie de cambios que han tenido directo impacto en las culturas y la autocomprensión que tienen estas de sí-mismas $y$ de otras culturas. Consecuencias directas de dichos cambios son una progresiva transformación del curso de la vida, sus periodos, transiciones y trayectos (Elder, 1994; Kohli,
1998). El periodo juvenil es uno de los periodos en los que de forma más dramática se ha vivido un proceso de reestructuración, que supone una progresiva biografización y relativa desestandarización del curso de la vida, así como con la formación de culturas juveniles que rompen con límites etarios (Pertersen y Peters, 1983; Büchner, 1995; Epstein, 1998; Win y White, 1997). Por otro lado, el trabajo cotidiano sobre la identidad no es un tema exclusivo de la fase adolescente sino que esta es una actividad permanente. La independencia respecto de la familia así como la autonomización de los bosquejos de vida, tampoco pueden ser homogenizados en tanto experiencias de profunda crisis o como procesos ubicables en un momento determinado de la fase juvenil. Por el contrario, es posible encontrar diferentes estrategias de enfrentamiento a estas tareas de desarrollo (Wyn y White, 1997 y Meuss, 1992).

El presente artículo pretende indagar en las nociones que poseen adultos de dos condiciones institucionales diferentes, acerca del periodo juvenil y el papel que juegan los medios de comunicación de masas en la juventud actual, para lo cual se definieron las siguientes preguntas como problema de investigación: ¿cuáles son las representaciones de la juventud construidas por adultos de dos condiciones socio-institucionales?; ¿qué papel juega la representación sobre las relaciones intergeneracionales en la comprensión de la juventud actual? Asimismo se planteó sobre el papel que se le atribuye a los medios de comunicación en la comprensión de la juventud y cuáles son las semejanzas y diferencias existentes entre las representaciones producidas por los adultos de diferentes condiciones sociales. Este artículo es el producto de un estudio llevado a cabo por los autores entre 2003 y 2005, en el marco del Instituto de Investigaciones Psicológicas de la Universidad de Costa Rica.

De esta manera el estudio se basó tanto en los modelos psicosociales para el estudio de las cogniciones sociales como en las aproximaciones fenomenológicas a las representaciones sociales. Se apeló a las teorías de los esquemas cognoscitivos entendidos como sistemas de representaciones acerca de situaciones, personas, cosas o estrategias de acción (Fiske y 
Taylor, 1991; Páez, Márquez e Insúa, 1998). En particular fueron relevantes los esquemas de personas o grupos que permitieron la comprensión del comportamiento de individuos recurriendo a su categorización, de conformidad con modelos socialmente producidos. Igualmente interesó analizar esquemas heurísticos atributivos que permitieran explicar la conducta de las personas, así como el impacto de situaciones sociales en las características de grupos sociales $o$ individuos concretos. La formación de esquemas se comprendió en el marco del modelo conexionista de formación de cogniciones sociales, partiendo del supuesto que en la interacción sujeto-ambiente social se activan nodos que de forma especializada o de forma ad-hoc permiten el procesamiento y comprensión de la información (Smith y Queller, 2003). La activación de estos nodos responde tanto a los llamados procesos top-down como botton-up que definen la construcción de conocimiento y la comprensión del mundo social, en este caso de grupos sociales, su interacción y la explicación de su comportamiento. Ahora bien, es importante dejar claro que los sistemas de representaciones aunque son producto del procesamiento individual tienen su origen en las comunicaciones sociales tanto en las relaciones interpersonales como en la comunicación de masas, $y$ en ese sentido son construcciones colectivas. La teoría de las Representaciones Sociales (Flick, 1995; Farr y Moscovici, 1984) aporta importantes elementos para la comprensión de la génesis social de las representaciones. Se trata de un conjunto de saberes, valores y formas de actuar proveídos por la tradición, los discursos científicos o religiosos que permiten interpretar el mundo social, los sucesos que allí acontecen y a las personas y grupos que lo integran.

Con las fuentes que teníamos disponibles al realizar el estudio se encontró que no solo al nivel nacional, sino también al internacional es escasa la investigación sobre el tema. En este contexto cabe destacar sin embargo, la investigación de Christy Buchanan y colaboradores (Buchanan, 2003 y Buchanan y Holmbeck, 1998) que se ha dirigido al estudio de las creencias de adultos y madres en particular en torno a la adolescencia. Los resultados dan cuenta de la tendencia a realizar una descripción básica- mente negativa de la adolescencia, como periodo tormentoso de riesgos y en el cual padres e hijos deben mantenerse más cercanos. Esta descripción varía si se trata de uno de los hijos o de una creencia generalizada de la juventud, en el primer caso se atiende a la experiencia concreta, dando cuenta de las particularidades que varían de situación en situación. Igualmente se recurre a una evaluación de las características atribuidas a los adolescentes y no solo a una descripción de rasgos. Esta evaluación negativa puede explicarse en el marco de los cambios históricos que se han dado en la infancia y la adolescencia en los últimos cincuenta años (Corsaro, 1997; Büchner, du Bois-Reymond, Ecarius, Krüger, 1998) y que se dirigen a una progresiva autonomía de la adolescencia en tanto periodo del curso de la vida, pero también en tanto grupo socio-cultural. Este proceso lleva aparejado tanto la construcción históricosocial de culturas etarias, que aunque relacionadas entre sí socio-estructuralmente, llevan a la formación de mundos de la vida con relativa independencia, promoviendo marcos sociocognoscitivos y comunicativos que favorecen formas específicas de evaluación y el enjuiciamiento intergeneracional vinculados con la estereotipia y el conflicto entre los diferentes grupos etarios.

\section{MÉTODO}

El enfoque metodológico que orientó la presente investigación fue el llamado "análisis de modelos de interpretación cultural”. Según, Lüders (1991) el interés general de este enfoque es el estudio de la experiencia cotidiana, es decir, la determinación de esquemas culturales e históricos para la interpretación de la realidad. Los modelos de interpretación se refieren a estructuras de sentido cultural, que tienen el carácter de reglas que regulan tanto la comprensión del mundo social como la acción sobre este. Según Oevermann (1973), "bajo la noción de modelos de interpretación no deben entenderse opiniones aisladas o actitudes respecto a un determinado objeto de acción, sino más bien se trata de contextos de argumentación que se estructuran según reglas de consistencia” (p. 3). 


\section{SUJETOS}

La definición de los sujetos de investigación se llevó a cabo fuera de la noción probabilística de muestra. Como señala Canales y Peinado (1995), interesó más bien definir los tipos sociales con los que se van a trabajar, entendidos como diferentes formas discursivas que dan cuenta de variaciones semánticas y pragmáticas diversas en torno a la juventud. En esa misma línea Gaskell (2000) plantea que en la investigación cualitativa las muestras no se orientan a la selección de personas sino a la definición de dimensiones sociales relevantes para el estudio. En concreto, la presente investigación tomó como espacio social general a la provincia de San José, zona en la que se evidencia un gran flujo $y$ diversificación socio-cultural, aspecto determinante en la comprensión de la juventud. La representación de la juventud desde los adultos en tanto grupo se abordó como mediatizadas por la situación social (soziale lage, Hradil, 1987), es decir, por las dimensiones que definen la calidad de las condiciones de vida $y$ la posibilidad de alcanzar las metas vitales propuestas por los individuos o grupos, las cuales varían socio-históricamente. Para efectos de la presente investigación se analizaron dos situaciones sociales definidas teóricamente por las diferencias en las posibilidades materiales, infraestructurales y sociales de realización de las metas vitales: a una la llamaremos situación social profesional-gerencial (SSP) y a la otra subalterna-asalariada (SSS), retomando pero de forma modificada la clasificación de Lobo y Robert (1996). La primera situación está constituida por familias cuyos miembros son personas altamente calificadas, que se dedican al ejercicio liberal de la profesión, se desempeñan en puestos de mando o de alto prestigio social. La segunda situación está compuesta por familias cuyos miembros son empleados del sector público o privado con estudios secundarios o técnicos, en puestos medios o bajos de poco reconocimiento social. Con cada "tipo" se realizaron dos grupos de discusión, número mínimo necesario para efectos de comparación de los análisis y de confiabilidad (Gaskell, 2000). En el caso de los grupos de situaciones sociales asalariadas subalternas, estos se contactaron en colegios públicos, mientras que los grupos de situaciones sociales profesionales-gerenciales en colegios privados. En cada institución se abordó un grupo de padres de familia $y$ un grupo de docentes de secundaria compuestos por hombres y mujeres.

\section{PROCEDIMIENTOS DE RECOLECCIÓN}

Se recurrió al método de los grupos de discusión (Canales y Peinado, 1995; Lamnek, 1998; Valles, 1997; Gaskell, 2000), procurando un enfoque interaccionista de esta técnica (Niessen, 1979 y Lunt $y$ Livingstone, 1996), el cual da importancia a la homogeneidad y la cercanía social en la composición de los grupos, con la intención de reproducir, en alguna medida, las conversaciones y la producción cotidiana de sentido. Se escogieron personas que se conocieran entre sí o que compartieran espacios sociales-institucionales comunes, específicamente se trabajó con docentes de un mismo colegio y con madres/padres de familia cuyos hijos estuviesen en una misma aula. Los grupos de discusión se realizaron además en los colegios donde se contactaron a los docentes y padres. Estas medidas aseguraron que el marco social de interacción, así como las reglas básicas de conversación y de atribución de sentido fueran compartidas por los grupos.

Se elaboró una guía para la realización de los grupos. Las preguntas que conformaron dicha guía debían promover la generación de teorías implícitas o teorías ingenuas sobre la juventud (Bruner y Tagiurir, 1956), que a su vez permitieran generar categorizaciones sociales $y$ atribuciones de causalidad o intención. Para esto la contrastación vía identidad social, es decir, apelar a mecanismos de comparación intergrupal, en este caso intergeneracional, fue fundamental.

Las siguientes fases comprendieron la recolección de datos:

a) Confección de las guías de discusión y los materiales asociados: en esta fase se definieron los temas $y$ preguntas generadores del grupo de discusión. Además se elaboró el material estímulo, que se introduciría al inicio del estudio. 
b) Realización del grupo de discusión: cada grupo de discusión tuvo una duración promedio de 50 minutos. La moderación del grupo estuvo compuesta por un coordinador $y$ un o una asistente. Se promovió la producción argumentativa sobre los temas de discusión, fomentando la participación de todos los miembros. La discusión se registró tanto en audio como en vídeo, esto con el objetivo de ampliar las posibilidades de procesamiento analítico de los resultados. Con el fin de reducir el efecto de artificialidad de la situación, los grupos de discusión se llevaron a cabo en los colegios a los que están vinculados los participantes.

c) Protocolización de la información: aquí se recurrió a una trascripción comentada (Mayring, 1993), en la que junto con la respectiva versión textual de la información se recurre tanto al uso de un sistema de códigos para el registro de aspectos tales como pausas, entonaciones, usos lingüísticos particulares como a la inclusión de observaciones situacionales producto de las anotaciones del asistente o que aparecen al observar los vídeos.

\section{PROCEDIMIENTOS DE ANÁLISIS}

Se recurrió a un análisis de contenido cualitativo de tipo estructurante (Mayring, 1993), específicamente al modelo propuesto por la Grounded Theory.

Strauss y Corbin (1998) han sistematizado un procedimiento para la realización de esta propuesta de análisis de contenido, la cual sirvió de base para la realización de la presente investigación. Se pueden distinguir las siguientes fases: 1) codificación abierta, 2) codificación axial y 3) codificación selectiva. En la primera fase se hace una identificación de las categorías básicas sus propiedades $y$ dimensiones, para lo cual se parafrasean pasajes o seleccionan ejemplos claves. A la par de la definición de las categorías, se van sistematizando ideas, interpretaciones o problemas, en la forma de memorandos, para el posterior análisis. En la segunda fase, se procede a una primera integración de la información determinando las relaciones estructurales entre categorías y subcategorías, sus dimensiones y propiedades. En la tercera fase se procede a la reconstrucción del modelo comprensivo que está a la base de las estructuras o procesos identificados en la fase anterior.

\section{VALIDEZ}

Se recurrió a un criterio de validez interna que es parte intrínseca del método de comparación constante (Glaser y Strauss, 1998 y Steinke, 1999). Por un lado, se recurrió tanto a la realización de análisis repetidos y comparados por parte del equipo investigador, como al criterio de saturación de información para cada categoría y sus relaciones, corroborando que la interpretación mantuviera su fundamento con los datos. Por otro lado, se procedió a la comparación de los resultados entre los grupos correspondientes a una misma condición. En los casos que así lo requirieron se procedió a una revisión de las relaciones estructurales encontradas entre las categorías, manteniéndose nuevamente aquellas que son respaldadas ampliamente por los datos.

\section{RESULTADOS}

A continuación se presentan los resultados que resumen la codificación axial, de los grupos de padres $y$ de docentes por separado, estableciendo comparaciones al interior de cada grupo entre las dos situaciones sociales estudiadas SSS y SSP. Junto con la descripción de los resultados se incorporarán pasajes, cuyo objetivo es la ilustración del material base a partir del cual surge la codificación. Consideramos que la codificación en la Grounded Theory se construye a partir de los protocolos como totalidad, así que el recurso a la ejemplificación, aunque muy utilizado, no es una estrategia adecuada para exponer los análisis obtenidos. Ideal sería adjuntar los protocolos, pero obviamente por razones de espacio eso no es posible. Los interesados en obtenerlos con fines de investigación pueden comunicarse con los autores. 
COMPARACIÓN GRUPOS

DE DISCUSIÓN PADRES SSS Y SSP

\section{ESQUEMA GRUPO DE JÓVENES}

\subsection{FORMACIÓN DE IMPRESIONES}

En una y otra situación social se consideran rasgos inherentes al periodo juvenil, tales como la tensión entre la búsqueda de identidad, la necesidad de diferenciación de los padres y la búsqueda de aceptación por parte del grupo de pares. Ahora bien en sss se resalta además el buscar un estilo propio que los diferencie de otras generaciones y que se expresa a través de la moda o en las actividades que realizan. Mientras que en la SSP se considera como un distintivo adicional, la tendencia a la rebeldía y el desafío a la autoridad.

Ambos grupos caracterizan a los jóvenes actuales como irrespetuosos de los padres, de las reglas impuestas y carentes de límites, atribuyéndose derechos pero no deberes. Se señala también que viven despreocupados, interesados en el presente $y$ no en el futuro.

\section{FRAGMENTO DE UN GRUPO DE DISCUSIÓN CON PADRES Y MADRES DE SSS (*)}

Moderadora: (?) cómo ven ustedes a la juventud actual (,) cómo la describirían

$E A$ : que pregunta más atrevida verdad

$E C$ : yo siento que no respetan los límites que se les establecen (,) o que hay padres que no marcan límites (,) y entonces ellos hacen lo que quieran entonces

$E A$ : es que con el aspecto de la juventud actual hay un problema (,) ehh quizá las que estamos en la casa (,) tenemos un poco más de acceso a ellos y podemos marcar más límites (,) mientras que los padres (,) que desgraciadamente tienen que trabajar (,) que llegan después de las cuatro o cinco de la tarde (,) el muchacho ahí hace lo que quiera (,) ellos se van involucrando (,) y se van complicando mucho más actualmente (,) o sea se dejan llevar más por las masas (,) por los otros compañeros (,) porque no hay no hay una persona ahí que les ponga un freno en ese momento (") ese es el problema

Moderadora: (?) alguna otra razón de por qué los jóvenes son así

$E C$ : yo pienso que (,) ha habido un gran deterioro en lo que es (,) son los principios espirituales sobre todo (,) este ehh (,) los muchachos (,) no sé (.) hay muchas familias y ahí no me incluyo yo (,) que no orientan a sus hijos espiritualmente (,) son familias que simplemente (,) traen los hijos al mundo y no sé si algún día les dirán si existe dios o no (,) pero los chiquillos crecen (,) no van ni a un culto ni a una misa ni a ningún lado (,) entonces este ehh (,) pues eso provoca que haya cierta ehh (,) desorientación en el muchacho (,) en el sentido de que de que no tiene un dios (,) un temor a dios (,) y cree que todo está bien (,) eso por un lado (,) y después yo si creo que los padres (,) aún cuando se trabaja (,) tenemos que ser conscientes de que primero somos padres (,) antes que empleado que trabajador (,) y tener control aún (,) de de los hijos dentro del trabajo de uno (,) uno necesita estar expectante de dónde están (,) con quién están (,) este cuál es la casa a dónde van a ir a esa fiesta (,) quién está en esa casa (,) quiénes son los papás de ese chiquito (,) cuáles son sus costumbres (,) y hay muchos papás (,) como quien dice (,) por quitarse los chiquillos de encima (,) se despreocupan de todo eso (,) que ellos se vayan a las fiestas o de todo

(*) Los participantes se identifican con letras para mantener su anonimato

Los padres de los grupos sSS se refieren de forma predominante a características morales de los jóvenes resaltando los elementos negativos, riesgosos y críticos de esta generación. Se considera que son jóvenes que han roto los vínculos de autoridad paterna y presentan un estilo de vida contrario al familiar, separándose de los valores centrados en el esfuerzo, prefiriendo la vía fácil. No les interesa el estudio, por el contrario, se burlan de los que están preocupados por ello y el reprobar es algo normal. La generación joven actual está guiada por los valores materiales, expresados a través de la predilección por las marcas o la burla de los que menos tiene.

Los jóvenes se van a percibir como perdidos, apresurados, irreflexivos y orientados a probar lo que les perjudica. Son una generación con mayores libertades, entendidas estas en términos de libertinaje, es decir, fuman o mantienen encuentros amorosos de manera pública. La sexualidad es experimentada y aprendida de forma abierta, en los colegios los embarazos adolescentes logran integrarse en la cotidianidad 
estudiantil, percibiéndose como algo normal e incluso positivo. Esta última tendencia es vista de forma negativa, pues puede promover la imitación por parte de otras jóvenes.

Ahora bien, en esta situación se pueden identificar dos polos en la descripción de la juventud, por un lado están los esforzados, maduros, con personalidad, que siguen los mismos patrones de comportamiento de la juventud anterior $y$ por otro lado, los vagos $y$ despreocupados.

Por su parte, las madres $y$ padres de los grupos SSP se refieren de manera dominante a rasgos psicológicos asociados a los jóvenes, señalando los riesgos pero también los alcances o cualidades positivas atribuidas comparativamente a la presente generación. Así, la búsqueda de identidad $y$ la necesidad de diferenciación de los padres se vislumbra desde dos perspectivas: como ligada a la orientación vital al grupo de pares o en una diferenciación funcional que mantiene la dependencia con los padres. Se trata a la vez de una juventud mediática y estructurada por la industria cultural, que dicta estilos y modas. Se considera como un logro generacional el que sean más inteligentes, informados y con mayores competencias comunicativas en las relaciones que establecen, lo que favorece incluso la relación entre padres e hijos. Pero se observa con preocupación el hecho de que estén "madurando" más rápido en comparación con las anteriores generaciones. A la par de estas descripciones, los grupos que corresponden a la SSP no descartan la caracterización de tipo moral. Por el contrario, la incorporan con preocupación considerando que los rasgos positivos asociados a los jóvenes pueden tener como consecuencia la falta de límites y de respeto hacia los padres.

La presente generación no se guía por principios morales, sino más bien están preocupados por lo material, tener lo suficiente para consumir. El trabajo, el estudio y la elección de pareja están influenciados por la realización material, aunque se reconoce que esto último no es exclusivo de los jóvenes. Además el matrimonio deja de ser una meta y se prefiere vivir con una pareja.

De igual manera la tendencia a la experimentación puede llevar a riesgos como el acceso a drogas. En este sentido, se distingue una juventud problemática de otra no problemática, así como una juventud rural, tradicional de una juventud citadina, que no logra satisfacerse con

Fragmento de un grupo de discusión con padres y madres de SSP (*)

Moderadora: y en general (?) cómo ven ustedes a la juventud actual digamos cómo la describirían (,)

$B$ : (?) cómo la describirían (,) bueno yo si siento que la juventud actual es muy inteligente (,) al tener tanta información (,) están tan expuestos digamos a una serie de cosas (,) que digamos nosotros no estuvimos tan expuestos (,) para bien o para mal verdad (,) porque tiene sus sus aspectos positivos y sus aspectos negativos (,) pero yo siento que sí son mucho más inteligentes más analíticos (,) ehh tienen más elementos de juicio para poder este (,) este opinar verdad (,) es inevitable (,) y de hecho te cuestionan y te llevan $y$ te traen y $y$ si

$D$ : porque es una cuestión pienso yo de que (,) en el tiempo de antes uno no le podía decir al papá o a la mamá nada (,) es decir ahora uno se los permite (,) y no se cuenta verdad (,) antes no (,) dios guarde yo le dijera a mi papá como me dicen mis hijas (.) estás loca vos no seas tan ridícula (.) así le dicen a uno (,) y yo cuando yo le dijera a mi papá una cosa (,) me volvía la cara para el otro lado

$B$ : pero es que eso no necesariamente era positivo porque hasta cierto punto están expresando su (,) su (,) su sentir verdad (,) yo siento que eso (,) a pesar de que a uno como padre no le llama mucho la atención (,) no le sea no le sea lo más agradable que un hijo le puede decir (.) estás loca como se te puede ocurrir qué ridícula (,) pero pero están expresándose cierto

$D$ : no si y eso es bueno (,) yo más bien lo veo positivo (,) o sea porque ellos te dicen con criterio y te defienden un punto que para vos puede ser bueno o malo pero (,) lo están defendiendo con capacidad porque averiguaron solos (,) por cualquier medio verdad

(*) Los participantes son identificados con letras para mantener su anonimato 
nada y es apresurada. Así, citadino y problemático se ponen en asociación. Esta generación se caracteriza por la anomia o la confusión moral. Los jóvenes son catalogados como egocéntricos, aunque si les preguntan, tienen una opinión propia sobre los problemas sociales.

\subsection{ATRIBUCIÓN DE CAUSALIDAD}

Cada situación social atribuye diferentes explicaciones que permiten entender el comportamiento juvenil. En ambos grupos las atribuciones realizadas no se refieren a causas intrínsecas asociadas a la juventud, sino más bien a factores situacionales, siendo el más relevante la familia, seguido, por los medios de comunicación y la industria cultural, así como el grupo de pares. Respecto a la familia las explicaciones se focalizan en la falta de imposición de límites por parte de los padres, así como en el impacto negativo en la crianza de los hijos producto de que ambos padres trabajan y del divorcio. Esta última condición lleva a que los jóvenes recurran a los pares con los consecuentes riesgos que esto podría implicar. El grupo de pares se considera como otro factor explicativo del actuar adolescente, entendido en términos de influjo que riñe con las normas $y$ costumbres familiares, al ofrecer modas, estilos o comportamientos riesgosos para la salud o la moral (drogas, sexo). Los grupos de la SSP, señalan que dicho influjo conduce a una reducción de la autoestima en los jóvenes al depender de los otros para definir su identidad. Acerca del impacto de la industria cultural, los grupos de ambas situaciones señalan atribuciones asociadas sobre todo a la televisión y en segundo lugar a la Internet, mostrándose críticos ante la saturación de contenidos ligados a sexo, $y$ violencia.

Por su parte, en la SSs se crítica el cambio en la relación padres-hijos y los estilos de disciplinamiento. Se considera que los padres ya no ejercen una función de autoridad y de guía espiritual, lo que se expresa en un descuido en la crianza y la pérdida de control sobre los hijos. Los padres depositan la formación de sus hijos en la escuela; sin embargo, descalifican a la institución escolar, la disciplina institucional y formación de los educadores. Esto es explicado como una estrategia equivocada de padres ausentes que tratan de acercarse a sus hijos.

Estas transformaciones se refieren a condiciones sociales $y$ de estructuración familiar también cambiantes y que atentan contra la familia, como el trabajo de las madres, que dejan a los hijos desprotegidos, al ser estas las únicas que se hacían cargo de los hijos, ya que los padres siempre han estado ausentes. La televisión se convierte en agente socializador, sustituyendo a los padres, así los modelos paternos son reemplazados por personajes mediáticos riesgosos.

En los grupos de la SSP se presenta una posición que explica las características de los jóvenes como producto de la llamada "democratización" de la familia, que considera a los hijos con derechos. Esto ha llevado a un temor por ejercer la autoridad paterna. Al respecto se consideran dos posiciones: la primera señala que los límites deben respetar la individualidad, mientras que la segunda considera que la individualidad es un riesgo.

En lo referente al efecto de los medios, los grupos de la SSS perciben que el uso desde edades tempranas de los nuevos medios como la computadora, los juegos y el teléfono celular hace a los jóvenes madurar más temprano, llevándolos a sentirse adultos, capaces de realizar actividades o tomar decisiones para las cuales no están preparados.

La SSS, adiciona otros factores explicativos relacionados con el consumismo imperante de la sociedad actual, la sensación de mayor peligrosidad y el aumento de la permisividad social que conlleva al libertinaje.

En la SSP se considera que la televisión es sinónimo de sexo, homosexualidad, consumismo, agresión y que junto a otros medios promueve la homogeneidad. Igualmente la industria cultural ofrece ahora alternativas para "descarga de adrenalina" que pueden tener implicaciones negativas, al promover la experimentación en actividades riesgosas. 
COMPARACIÓN GRUPOS DE DISCUSIÓN DOCENTES SSS Y SSP

\section{ESQUEMA DE LA GENERACIÓN JOVEN ACTUAL}

\subsection{FORMACIÓN DE IMPRESIONES}

Cuando se describe a la generación joven actual se recurre a la enumeración de rasgos de dos tipos, los considerados inherentes al periodo del ciclo vital, es decir, propios de la juventud independientemente de la generación y los rasgos propios de la juventud actual que marcan una clara diferencia con la generación anterior.

Con respecto a los aspectos inherentes, los grupos de ambas situaciones consideran la tendencia a la diferenciación respecto a los padres $y$ la tensión padres e hijos como intrínseco a la experiencia de la juventud, expresado en la percepción negativa de la forma de actuar y de vestir del joven. En la SSS se vislumbra como característico de la juventud, el tener mucha energía acumulada que debe ser liberada. Dicha energía puede tener repercusiones positivas o negativas dependiendo como se canalice. Asimismo se agrega a las particularidades una tendencia a la rebeldía, con respecto a los adultos y sus normas.

En la SSP se concibe la adolescencia como un proceso de cambio. Además una posición visualiza que tanto el comportamiento adolescente, como la diferenciación con respecto a los padres, es algo pasajero que finaliza cuando los jóvenes cumplen la mayoría de edad y comienzan a trabajar.
Como rasgos característicos del grupo joven actual ambos grupos enumeran una serie de elementos que se le asocian. Se considera que la juventud actual experimenta una tensión entre la búsqueda y la definición de la identidad y la masificación, entre aislarse y pertenecer, de seguir un mismo patrón de conducta asociado al grupo de pares y a la industria cultural o resistirse a seguir esos modelos. El grupo de pares promueve la homogeneidad en el lenguaje y la falta de identidad, la industria cultural difunde antivalores, la imitación de estilos (tatuajes) y la actividad sexual.

Dentro de la búsqueda de una identidad propia y la diferenciación respecto a los padres, los grupos en esta situación consideran que los jóvenes recurren al propio cuerpo como parte de un proceso de apropiación y de afirmación de sí mismos, a la vez que como forma de revelarse contra el statu quo; de allí que el uso de los piercing y los tatuajes, sean considerados como una forma de auto-flagelo o auto-agresión.

En el plano valorativo, se considera a la juventud actual como conformista, orientada a la comodidad, descuidando los estudios y dando cabida a salidas fáciles para ganarse la vida, muchas de estas alternativas son aportadas por la televisión, por ejemplo robar. En esa misma línea, son también un grupo sin expectativas acerca del futuro personal, sin objetivos, esperanzas, ni aspiraciones. Se orientan por las apariencias, lo que se expresa por su interés en la moda, las marcas y el exterior de las personas.

La SSS plantea que la búsqueda de aceptación del grupo de pares los lleva a alejarse de las formas de convivencia más sanas, que no

FRAGMENTO DE UN GRUPO DE DISCUSIÓN CON DOCENTES DE UN COLEGIO DE SSP (*)

Moderadora: entonces (?) cómo ven ustedes a la juventud actual (,) (?) cómo la describirían

$D$ : yo este (,) a la mayoría de los adolescentes (,) bueno lo que veo aquí (,) yo los veo un poco desorientados (,) y generalmente (?) qué es lo que se les transmite (,) que para ellos hay muy pocas oportunidades (,) o que ellos son una generación que ya cuando van a comenzar a ser adultos (,) no tienen oportunidades para trabajar (,) no (,) hay espacios en los trabajos (,) (?) y para qué van a estudiar equis carrera (,) que ya está saturada (,) la la la demanda digamos (,) entonces ya no hay (,) (.),(...) para salarios de trabajo (,) entonces muchos que se sienten (,) presionados o como sin mundo (,) yo creo que si ehh hay futuro para ellos (,) si nos ponemos a pensar (,) muchas veces pero no (,) un trabajo de la Universidad Nacional (,) o en la OEA (,) en donde destacaron excelentes muchachos (,) y ellos participando y su tiempo y todo (,) porque es muy lindo $y(),.(.$.$) si tienen futuro y$ (,) si se pueden abrir camino pero (,) hay que saberlos orientar (.) pero desgraciadamente (,) los medios de comunicación colectiva transmiten todo (,)

(*) Los participantes son identificados con letras para mantener su anonimato 
son precisamente aportadas por dichos grupos. Se considera que los jóvenes disfrutan quebrantando las reglas.

Por otro lado, se recurre a la violencia como forma de relacionarse con los pares, sea tanto para agredir expresamente o a modo de expresar cariño.

Además se posiciona como una generación sin valores, cuyos líderes son los irrespetuosos, imperando la "ley del más fuerte". De igual modo se le considera un grupo etario orientado a la experimentación, el sexo temprano y con más acceso a información que anteriores generaciones.

En esta situación se pueden distinguir, sin embargo, dos posiciones: aquellos que se orientan a seguir a la familia $y$ sus normas $y$ aquellos que se orientan al grupo de pares. Los primeros son jóvenes calificados como correctos, ordenados, disciplinados, honestos, respetuosos, que van a fiestas y no hacen nada indebido y provienen de buenas familias. Están guiados por los valores de los padres orientándose al respeto, la responsabilidad y el trabajo. Los jóvenes de zonas rurales son percibidos como pertenecientes a este grupo. Los jóvenes orientados al grupo de pares corresponden a las características señaladas con anterioridad.
Los grupos de la SSP asocian también a la búsqueda de identidad y la diferenciación respecto a la familia, el imitar modelos y estilos de vida juveniles provenientes de los Estados Unidos. Esto se entiende además en el marco de la necesidad de adaptación al grupo de pares que los lleva a imitar una moda particular y a verse presionados a la integración como alternativa para ser aceptados grupalmente.

Existen otros rasgos de tipo psicosocial asociados a la juventud actual, como la soledad, la desorientación, la necesidad de llamar la atención, la falta de compromiso en las relaciones interpersonales. Además se les describe como fantasiosos e idealistas, lo que los hace más proclives a ser influenciados por factores externos. Relacionado con lo anterior se plantean dos posiciones, una visualiza una generación más permeable a presiones externas proveniente de los medios o el grupo de pares. La otra posición plantea que hay un tipo de jóvenes que no imitan estos modelos, que se muestran resistentes al influjo proveniente de la cultura estadounidense.

Por último, se señala que son una generación preocupada por su futuro laboral $y$ formativo, dado la incertidumbre que existe a este nivel.

FRAGMENTO DE UN GRUPO DE DISCUSIÓN CON DOCENTES DE UN COLEGIO DE SSS

Moderadora: a partir de lo que ustedes han dicho hasta ahora digamos sobre la juventud (?) cómo describirían ustedes la juventud actual

A: bueno yo estaba tomando aquí unos apuntes de algunas ideas que me estaban viniendo y y yo considero que la juventud actual es una juventud (, ) si bien es cierto es más problemática que la juventud de antes (,) es una juventud que le ha tocado vivir en una época más difícil porque es una época de mayores competencias digámoslo así y esto se debe a esta palabrita que los profesores de estudios sociales conocen muy bien (,) que es la globalización (,) la globalización con la apertura de mercados la ruptura de fronteras digámoslo así hace que todos los pueblos vayan perdiendo las identidades (,) entonces de alguna manera tienen que servir los subgrupos los grupos para buscar sus propias identi identificación (,) ahora yo a veces me pongo a ver de la propaganda veámoslo así con cosas muy sencillas (.) toda la mayoría de la propaganda que hay sea por radio televisión internet lo que sea es propaganda que está dirigida a los jóvenes (?) por qué (,) porque los jóvenes no tienen jamás la madurez como para reflexionar como para analizar las cosas que les están dando (...) (?) cuándo es que aprende uno a fumar (,) cuando es un adolescente porque es sumamente fácil entonces la propaganda sobre los cigarrillos está dirigida a los adolescentes porque ahí es dónde aprenden a fumar (,) un adulto muy difícil que aprenda a fumar ehh que se trague digamos ehh el gancho ese el amarre que tienen los medios publicitarios para vender un producto entonces yo yo considero que es eso (,) y a raíz de estos medios masivos de comunicación de este rompimiento de fronteras de esta masificación de esta (,) desintegración familiar que se produce entonces lo que está entredicho es (,) la pérdida de los valores (.) valores éticos los valores morales los valores familiares ehh en eso digamos yo más o menos yo sí he notado eso

(*) Los participantes son identificados con letras para mantener su anonimato 


\subsection{ATRIBUCIÓN DE CAUSALIDAD}

Los grupos de ambas situaciones elaboran una serie de causas que explican la conducta $y$ formas de pensar de los adolescentes. Común en las dos situaciones es el señalar el hecho de que ambos padres trabajen, dando peso a la presencia de la figura materna en la crianza de los hijos y como su ausencia puede generar la aparición de comportamientos y valores no deseados por los padres. Otro factor explicativo vinculado con lo anterior radica en los cambios en la crianza de los hijos, tendientes a una mayor permisividad.

En la SSS se considera que en sociedades machistas la salida de la mujer del ámbito familiar y su incorporación profesional va en desmedro de la familia, descuidando la educación de los hijos y confiriendo a los jóvenes tareas que no les corresponden y que son función de los padres. Este descuido es precisamente el que conduce a que los jóvenes busquen integrarse e imitar al grupo de pares. Dentro de este mismo ámbito se presenta una posición que considera que la mujer se ve obligada a salir de la casa para satisfacer necesidades consumistas, lo que conduce a que se convierta en una "esclava" del trabajo extra e intrafamiliar, obteniendo la pérdida de valores al interior de la familia.

Asociado a los anteriores condicionantes de causalidad, la sss considera que en la actualidad los padres depositan la educación de hijos en la escuela, la iglesia o los medios. No obstante, los padres desautorizan la disciplina escolar, como una forma falsa de congraciarse con los hijos. Esto se relaciona con un estilo de crianza paterno extremadamente tolerante y permisivo. Aunado a esto los jóvenes se comportan con sus padres de manera desafiante (amenazan incluso con acusarlos al PANI), de tal modo que los padres y maestros no se consideran apoyados por la institucionalidad que protege a los adolescentes. Además se plantea que los padres complacen de forma material a sus hijos bajo la condición de que sean exitosos en los estudios, librándose de la responsabilidad de formarlos.

Otra posición dentro de esta situación, postula que en la actualidad muchos de los padres son adolescentes, viéndose expuestos a los mismos contenidos mediáticos y estilos de sus hijos lo que impide una educación adecuada.

En la SSP se considera que los hijos ahora están en manos de las empleadas domésticas. La falta de dedicación paterna, genera en los jóvenes falta de cariño y una sensación de soledad. Ante esto se recurre a la búsqueda de modelos de identificación, al consumo de drogas, alcohol y a las relaciones sexuales ya sea como formas de llamar la atención o como sustitutos del amor que no obtienen de sus padres.

En este contexto, al interior de la familia los padres no ejercen ningún control, incluso se considera que las futuras generaciones de padres tendrán mayores problemas para establecer límites sobre sus hijos. No existen modelos que guíen a sus hijos, pero estos tampoco respetan a los adultos. En el ámbito escolar, si los maestros disciplinan a los jóvenes pueden ser acusados de agresión. Como en la sss se considera aquí que los maestros no tienen el respaldo de los padres, viéndose temerosos de actuar o negando los problemas de sus hijos.

Asimismo se señala que las familias cuyos padres se preocupan por transmitir e inculcar valores, no van a ser afectados por mensajes mediáticos. Esto supone una familia donde la madre no trabaja fuera de casa y el padre trabaja dentro del horario escolar de sus hijos. Esta condición considerada ideal, promueve jóvenes "sanos y limpios".

Otra posición dentro de esta situación considera que los cambios en la familia no son nuevos. La generación actual recoge los cambios de generaciones anteriores, volviéndose más problemática, propia de padres que no concluyeron sus proyectos.

Otra causa fundamental del comportamiento de la juventud actual, es atribuida al impacto nocivo del mensaje mediático y de la industria cultural en general. Según los grupos de ambas situaciones, los medios transmiten conductas, estilos de vida y valores, que riñen con las formas de vida $y$ los valores familiares.

En la Sss se enumeran diferentes formas en las que actúan los medios de comunicación. Así se considera a los grupos de rock como promotores de antivalores $y$ patrones de conducta que deben ser evitados por los jóvenes, teniendo un papel transformador en las formas 
de comportamiento juvenil de las zonas rurales $y$ sus estilos de vida tradicionales. Sin embargo, una posición considera que hay visiones alternativas como el rock cristiano, pero son poco consideradas por los medios, favoreciendo las imágenes estereotipadas de los jóvenes. Junto con los grupos de rock, la internet y la pornografía son fuente de imitación de conductas "negativas" por parte de los jóvenes.

De igual modo se plantea que ante la ausencia de los padres y la desintegración familiar, la televisión se ha convertido en el principal agente socializador, ofreciendo violencia $y$ drogas como forma de experimentación. Desde esta perspectiva, los medios de comunicación son entendidos como formas de contaminación cultural de la sociedad y cuyas funciones informativas traen consigo consecuencias fundamentalmente negativas.

Por su parte, la SsP considera que el mensaje mediático está ligado al desprecio a la vida y la desesperanza como generación, coartando el espíritu de lucha propio de pasadas generaciones, a favor de la despreocupación y el mínimo esfuerzo. Se promueve la libertad sin control, el consumo de drogas y la preocupación por la apariencia externa (modas). La música es considerada no solo con contenidos más rebeldes que de otras generaciones, sino estéticamente más fea. Todo esto es visto como un efecto aculturante $y$ enajenante que trata de imponer los estilos de vida estadounidenses. Ante los mensajes mediáticos los jóvenes no son auténticos, imitando lo que allí se presenta.

A la par de la aculturación está el impacto de los mensajes noticiosos. Se plantea que el constante bombardeo de los medios sobre eventos mundiales obliga a los jóvenes a un cambio continuo para adaptarse a esas imágenes, lo que lleva a que no tengan una imagen estable del mundo. Además, justifica la agresión hacia los padres y se promueve el desacato a los límites paternos.

Una tercera causa en la comprensión del comportamiento adolescente actual, compartida por ambas situaciones es la percepción de un deterioro moral de la sociedad.

Al respecto, los grupos de la sss consideran que la sociedad costarricense se encuentra en un estado de decadencia moral que puede llevar al colapso. Los jóvenes no tienen posibilidad de decidir entre lo bueno y lo malo. Los valores de las generaciones anteriores son ajenos a ellos, son una generación dirigida a la tecnología. Esto se asocia con la percepción de que muchos adolescentes "queman etapas", teniendo que hacerse cargo de obligaciones porque los padres están ausentes.

Otro problema asociado está vinculado con las leyes que protegen a la adolescencia, pudiéndose ubicar dos posiciones contrapuestas. Una posición señala que a la juventud se les da derechos que no deberían tener o merecer, mientras que no se les asignan deberes. La otra posición señala que esos derechos de los jóvenes son legítimos, el problema radica en como los usan y la irresponsabilidad individual sobre estos.

En los grupos de la SSP, se plantea que la sociedad actual vive en un estado de libertinaje y anarquía. En ellos están implicados padres, políticos e iglesia, mientras que los medios se encargan de propagar esta situación.

A esta condición general se agrega el hecho que los políticos son corruptos, promueven fórmulas fáciles e inmorales que no implican esfuerzo. No obstante se considera igualmente problemático que la juventud se limite a criticar a los partidos políticos, sin dar alternativas beneficiando a los que tienen el poder.

Los grupos de la sss hacen referencia a factores contextuales como causantes del comportamiento juvenil actual, como el vivir encerrados en comunidades dormitorio, siendo la única alternativa el consumo de televisión y la música. Sin embargo, en comparación con la juventud anterior, el contexto actual ofrece mayores posibilidades de diversión.

Se percibe además, que la pobreza ahora es mayor, dentro de un contexto que lleva a los jóvenes a integrarse en bandas o barras como forma de sobresalir y de buscar su identidad.

Aunado a esto, las oportunidades laborales o de estudio se vuelven en contra de los jóvenes, al encontrarse con un mercado saturado o no poseer información al respecto. De la misma manera el cambio del colegio a la universidad no es asimilado por todos, lo que los lleva al desvío y la rebelión.

Los grupos de la SSP por su parte consideran como un factor explicativo de las características 
asociadas a la juventud actual la situación del sistema educativo al dedicarse solo a transmitir conocimientos. Por ejemplo, los exámenes de bachillerato fomentan el facilitismo en vez del valor del esfuerzo y los diagnósticos de déficit atencional son sinónimo de "alcahuetería".

\section{DISCUSIÓN}

En este apartado se realizará inicialmente una comparación entre los discursos generados por grupos de adultos en relación con la juventud. Seguidamente se abordarán los resultados encontrados a la luz de los aportes teóricos de investigaciones precedentes.

Considérese ahora los aspectos comunes $y$ los distintivos entre ambos grupos de adultos. Tanto los padres como los docentes distinguen rasgos inherentes al periodo juvenil como rasgos particulares propios de la generación joven actual.

Con respecto a los rasgos inherentes, ambos grupos consideran las relaciones intergeneracionales conflictivas como un aspecto propio de la juventud. Al respecto se plantea la existencia de una tensión básica entre padres e hijos, la tendencia a la diferenciación y el distanciamiento respecto a los padres, así como la tendencia a la rebeldía y el cuestionamiento de la autoridad. Junto con estos rasgos, en el grupo de padres también se incorpora la tendencia a la búsqueda de aceptación por parte de los pares. En los docentes se incorpora la concepción de la adolescencia como un periodo caracterizado por la acumulación de energía que debe ser liberada.

Estos rasgos inherentes se describen a su vez incorporando especificidades propias de la época actual. Para los grupos de padres, la diferenciación adolescente con respecto a los padres es vista dentro del marco de las tareas propias de este periodo del curso de la vida, que no llega a rivalizar con la dependencia paterna que se mantiene aún en dicho periodo. Los docentes ponen de relieve las consecuencias negativas de la orientación al grupo de pares propio de este proceso de diferenciación, para señalarlos como promotores de la falta de identidad y de la homogeneidad en el lenguaje, las formas de actuar y de la moda juvenil, contribuyendo así a la masificación, que limita la individualidad $y$ la formación de una identidad personal y que igualmente puede conducir al despliegue de conductas riesgosas en el ámbito de las drogas, la sexualidad o formas de conducta que riñen con los patrones adultos del buen comportamiento.

De igual modo, los docentes conciben que en el contexto de la masificación, una de las principales formas a las que recurren los jóvenes para expresar su singularidad es el uso de tatuajes o del piercing, la cual es concebida como una forma de auto-agresión, un medio errado de autoafirmación.

Es importante indicar que al hacer referencia a los rasgos del grupo juvenil actual se recurre de manera fundamental a una evaluación de tipo moral. Se le caracteriza básicamente como: despreocupado, sin expectativas de futuro o metas. Consideran que el grupo juvenil actual está orientado a la búsqueda de la vida fácil, la comodidad $y$ a la falta de esfuerzo, expresada en la falta de empeño en el estudio. Los jóvenes son vistos como irrespetuosos y con tendencia a quebrantar las reglas o límites impuestos. Lo que va a mostrar variaciones en los grupos de adultos es la forma en que se aborda esta evaluación y la importancia que se le denota. Por ejemplo, los padres se refieren de forma predominante a la falta de valores, al irrespeto a la autoridad y a los límites, criticando y postulando los riesgos de su carencia. Los docentes además de describir la generación actual como carente de valores, recurren a una dicotomización moral de los jóvenes, dividiéndolos en buenos y malos, resultando que los buenos son aquellos que siguen el patrón de comportamiento ideal esperado por los adultos.

Es común en los grupos de adultos estudiados, el considerar que la generación actual está guiada por valores materiales, expresados a través del interés por las marcas y las apariencias. Desde la perspectiva de los padres, estos valores llevan a los jóvenes a la discriminación entre los que tienen o no tienen, o a reducir el estudio y el trabajo a un medio para consumir y adquirir. Tal interés por lo económico modifica hasta la elección de pareja, aunque esto más que una distinción de la 
juventud es una característica de los tiempos modernos-actualidad.

La experimentación es otro elemento distintivo, disposición que puede ser riesgosa como el consumo de drogas y que los lleva a orientarse a ofrecimientos que los perjudican, los vuelve irreflexivos o los pierde.

En el ámbito de la sexualidad se considera que tanto esta generación joven como las políticas de la institución educativa promueven una vivencia abierta de la sexualidad que se manifiesta en el mantenimiento de relaciones sexuales tempranas, en los encuentros amorosos públicos como en la tolerancia respecto al embarazo en adolescentes.

La juventud actual es vista como una generación orientada a los medios, la industria cultural y los estilos juveniles. Los matices entre los padres $y$ docentes se dan en que los segundos establecen que la industria cultural se encarga de transmitir anti-valores y modelos dudosos.

Un elemento que marcó una diferencia importante dentro de los adultos, es lo planteado por los padres de la situación social profesional, ya que si bien señalan los riesgos asociados a los jóvenes, también rescatan sus cualidades o logros como generación por ejemplo el que sea más inteligente, informada y que posee mejores competencias comunicativas, lo que a su vez favorece la relación con los padres.

Tanto los grupos de docentes como los de padres-madres definen como factores explicativos de la conducta adolescente a la familia, la industria cultural y los medios de comunicación, así como al grupo de pares. En cuanto a la familia y formas de crianza se abordan aspectos comunes como la falta de límites, la presencia de mayor permisividad paterna, las repercusiones familiares de ambos padres al mundo laboral, la desintegración familiar producto del divorcio, por mencionar algunos. En el caso de los padres que trabajan se enfatiza la ausencia materna y como esto afecta el entorno familiar (problemas en la de crianza de los hijos, falta de valores y la exposición de estos a conductas de riesgo). En particular el ingreso de la mujer al mundo laboral extra-familiar, se considera que tiene consecuencias negativas ya que genera desprotección, falta de control y la pérdida de horizonte moral en los jóvenes, quedando a merced del grupo de pares o los medios de comunicación. Además los padres ausentes suelen depositar en terceros su función paterna (cuido, socialización, educación), sea en empleadas domésticas, la escuela, la iglesia o los medios de comunicación.

Otro factor explicativo es la tendencia generalizada de los padres actuales de congraciarse con los hijos como una forma de acercarse a ellos, ya sea por la vía material o a través de la defensa y desautorización de los docentes $\mathrm{u}$ otros adultos. Tal situación percibida por el grupo de docentes, se expresa como un estado de indefensión. En relación con lo anterior los grupos de adultos identifican un fenómeno de cambio consistente en una democratización al interior de las relaciones entre padres e hijos, cambio que es visto más bien como riesgoso. Coinciden en que los derechos otorgados a los hijos pueden convertirse en un problema que repercute directamente en la relación entre padres e hijos. Esto por cuanto los hijos se arrogan derechos que no tienen, como el de no aceptar los controles paternos y actuar sin recibir la autorización de los padres. De igual manera, los padres por temor de irrespetar a los hijos, han desarrollado un temor a ejercer la autoridad $o$ al disciplinamiento.

En el caso de los medios e industria cultural, ambos grupos de adultos apuntan los riesgos de los modelos $y$ valores adquiridos a través de los medios (TV; internet) y cómo estos van en contra de los principios morales y familiares. Los medios de comunicación se han convertido en un poderoso agente socializador, formador del pensamiento y los patrones de acción de los jóvenes. Se considera que los mensajes de los medios están saturados de sexo, violencia y desesperanza, siendo justamente estos contenidos, los que aparecen como un claro factor de riesgo para los jóvenes.

Los docentes por su lado, consideran que los medios de comunicación como un factor de aculturación, la exposición a modelos estadounidenses $y$ la influencia de aspectos como la música rock en los estilos de vida juvenil, estos cambios son apreciados por ejemplo en jóvenes de zonas rurales. Mientras que los padres apelan a una preocupación derivada de la exposición a 
los medios, enfatizando en el papel que juegan estos en la maduración temprana de los jóvenes, en las alternativas negativas ofrecidas como forma de descargar adrenalina $y$ la promoción mediática de la homogeneidad juvenil.

No obstante, dentro de la descripción que realizan los docentes se hace una distinción del joven rural, vislumbrándolo como orientado a la familia y a los valores paternos; separándolo así de otros adolescentes que se ven guiados hacia el grupo de pares.

De igual manera, los padres logran distinguir una juventud problemática de otra no problemática, así como una juventud rural, tradicional de una juventud citadina.

Otro aspecto retomado en ambos grupos de adultos es lo referente al grupo de pares, este es vislumbrado como un peligro, una compañía riesgosa que influencia a los jóvenes. Esto se expone con mayor claridad en los padres en donde se coloca a los pares en contraposición al orden familiar. Si bien al describir el proceso adolescente general, los padres concebían la integración al grupo de pares como algo connatural a la adolescencia, en las sociedades actuales esto es más bien sinónimo de peligro.

Los docentes plantean de forma más clara y extensa una compresión a partir del deterioro moral, apuntando la carencia de principios. Los docentes consideran que la actual generación se enfrentará con mayores problemas para ejercer en las futuras generaciones autoridad $y$ límites. Estas dificultades refieren a su vez a las características de la pasada generación (la de los padres), que se caracterizaba por la poca claridad de metas y proyectos inconclusos.

Los padres por su lado, junto con apelaciones al deterioro moral, mencionan factores contextuales como la peligrosidad social y el consumismo como elementos causales.

En el discurso de los adultos que participaron en el estudio es clara la sistematización de una serie de tareas de desarrollo y de procesos atribuibles a la adolescencia, que se han venido sosteniendo como constantes básicas de este periodo por la psicología del desarrollo. Ya Erikson uno de los fundadores de la psicología de la adolescencia actual, venía refiriéndose a la búsqueda de la independencia y la consolidación de la identidad como tareas fundamentales del adolescente, tareas que traían aparejado conflictos intergeneracionales o la alienación producto de la aceptación de ideologías extrafamiliares propias de los grupos de pares o los nuevos grupos de pertenencia (ver Erikson, 1972 y una revisión crítica de Wyn y White, 1997). De esta manera, las teorías implícitas sobre la adolescencia que manejan los grupos adultos estudiados, responden en buena medida a una asimilación de los discursos científicos propagados por los medios de comunicación y el sistema educativo. Dichos conocimientos funcionan como esquemas heurísticos que posibilitan explicar las acciones de acuerdo con un esquema estable $y$ rígido que permite la categorización de las conductas adolescentes dentro de tipos estereotipados de adolescencia, sobregeneralizando su comportamiento y formas de pensamiento.

Este esquema no solo permite organizar la información sino valorarla, aspecto que fundamenta las respuestas emocionales concomitantes que se expresan en el discurso adulto de preocupación, repudio o bien empatía.

En este punto es donde se encuentra una primera diferencia entre los grupos de adultos, diferencia que no la encontramos entre las situaciones sociales estudiadas, sino entre los grupos institucionales. Los y las docentes presentan esquemas mucho más rígidos y sobregeneralizadores que las madres y los padres, mediante la representación de una contradicción insalvable entre el grupo de pares y la familia, resultando el grupo de pares sinónimo de riesgo, mientras que la familia, específicamente la dependencia respecto a los padres y el seguimiento irrestricto de su autoridad, es visto como curso deseable hacia la adultez madura. La familia entendida dentro del modelo patriarcal, es la que permitiría el desarrollo de una identidad sana, los pares promueven lo opuesto, ofreciendo masificación y peligro. En el discurso de los padres, en cambio la integración al grupo de pares es un comportamiento esperable $y$ es parte del proceso de independencia respecto a los padres. Las conductas riesgosas pueden sucederse, pero no son la norma y el impulso hacia la independencia.

Ahora bien, común a ambos grupos institucionales son los modelos mentales homogenizantes, que no dan cuenta de la realidad actual 
de la juventud, la cual se caracteriza más bien por una clara diferenciación y fragmentación, ligados a estilos de vida, modelos de consumo mediático o a su vinculación política. Esta tendencia refiere a un proceso de globalización cultural de la juventud como ha sido expuesto por autores diversos (ver entre otros a Feixa, Molina y Alsinet, 2002; Ferchhoff, 1999; o Cubides, Laverde y Valderrama, 1998).

De los parámetros atributivos explicatorios de la conducta adolescente, devienen los fundamentos del juicio moral sobre dicho grupo, contenido eje sobre el cual se desarrolla el discurso adulto sobre este grupo etario. Nuevamente resulta claro que el elemento diferenciador de los grupos en estudio, en la presente investigación, es la adscripción institucional y no la situación social. Ambos grupos tienen como prototipo de convivencia intergeneracional el modelo familiar patriarcal tradicional. De allí que "la salida" de la madre del hogar al mundo laboral, el cambio en los patrones de parentaje y la integración de los medios de comunicación como factor socializador sea visto como una amenaza. Es decir, la modernización social y la globalización cultural son vistos como amenaza a un orden moral que pautaba las normas de comportamiento al interior de las generaciones y entre las generaciones. Desde la perspectiva de los grupos adultos estudiados, los padres ya no cumplen con su rol de autoridad y de transmisor cultural, de allí que los jóvenes sean percibidos como puestos a merced de una organización social sobre la que no se tiene control. Ante la pérdida del horizonte moral que guiaba las trayectorias de la infancia a la adultez, solo queda el caos moral, los estilos de vida no saludables y la desintegración social.

Hay un aspecto que con certeza señaló Erikson décadas atrás, $y$ es el hecho de que en el periodo juvenil queda plasmado con claridad el vínculo entre historia social e historia biográfica, en este periodo se escenifica de forma viva las transformaciones socio-culturales y el devenir de la sociedad como un todo.

Estas afirmaciones siguen siendo vigentes $y$ nos permiten dimensionar el discurso adulto sobre la juventud. De lo que estamos siendo testigos no es del caos moral o la desintegración cultural, más bien somos testigos y copartícipes de un proceso modernizador de diferenciación cultural, en el que procesos de autonomización reflexiva del curso de la vida al interior y fuera de la familia conviven con los determinantes socio-estructurales del acontecer biográfico. La sociedad organizada en torno a la familia pierde fundamento $y$ otros agentes culturales tienen un protagonismo más visible. Por supuesto, en este cambio ciertamente hay riesgos, pero también hay oportunidades.

\section{CONCLUSIONES}

A partir de los hallazgos encontrados es posible extraer una serie de elementos importantes para el análisis de la representación y la configuración de la juventud actual a través de la perspectiva de los adultos.

En el caso de los grupos de adultos estudiados se concibe la adolescencia como un periodo definido del ciclo vital claramente distinguible de la infancia y la adultez. En la representación construida se mezclan tanto los contenidos propios de la visión romántica de adolescencia como las nociones aportadas por la psicología del desarrollo. En la primera se hace referencia a la adolescencia como un periodo tormentoso y de crisis, en la segunda se hace énfasis en los fenómenos de separación, individuación, búsqueda de identidad, la orientación a los pares y los riesgos que consecuentemente pueden tener dichos acontecimientos.

Ahora bien, esta visión es construida desde la perspectiva familiar e incluso en tanto padres o madres, independientemente de que se haya tratado del grupo de docentes o el de padres. En ese sentido, es percibido como un periodo universal, generalizable del ciclo vital que se aplica tanto a su propia experiencia adolescente como al grupo joven actual. Pero igualmente se le concibe como un momento dentro del ciclo familiar, en el que los hijos y los padres establecen relaciones particulares, por lo general de tipo conflictiva y los hijos cambian sus conductas respecto a la familia, quedando cuestionadas las relaciones de autoridad $y$ de seguimiento de la normatividad familiar.

Esta constante presenta sin embargo, variaciones históricas entre una generación y 
otra, atribuible a una combinación de cambios contextuales y morales. Desde su perspectiva, la generación actual ha perdido el vínculo tradicional con la familia y con las figuras de autoridad, adquiriendo el conflicto generacional otro matiz. Ya no es solo un evento intrínseco de este periodo del curso de la vida, sino que adquiere carácter de crisis moral, sobre todo desde la perspectiva de los docentes. Una crisis moral que da cuenta de un cambio social visto con preocupación, ya que refleja un estado de anomia y de disolución de nexos sociales tradicionales. Esta percepción refiere a la valoración con reservas e incluso crítica de un proceso de modernización social que supone la diferenciación y pluralización de los períodos del curso de la vida, cada vez menos ligados a la reproducción de un patrón biográfico homogéneo. Este mismo proceso supone un cambio en las relaciones jerárquicas familiares pasando de un modelo autoritario propio de las relaciones entre padres e hijos a formas de crianza cada vez más permisivas $y$ dialogantes. Este cambio es visto de forma contradictoria por los padres, ya que no tienen claridad sobre la forma indicada de poner límites o ejercer control sobre los hijos, aunque se recoge los aspectos positivos de este cambio, que implican mayor comunicación, autonomía, capacidad de deliberación e información por parte de los jóvenes. Para los docentes el cambio es visto más bien como una amenaza para los padres y los mismos docentes, ya que atentan contra su autoridad y no permiten controlar, disciplinar y educar a un grupo etario que depende de la orientación adulta para su desarrollo, según las normas de comportamiento juvenil que suponen un hijo obediente, dedicado a los estudios, la familia y actividades recreativas como los deportes.

Son claras las diferencias en el plano evaluativo entre padres $y$ docentes. Los docentes hacen una valoración más negativa e incluso prejuiciosa y agresiva de la juventud actual. Esto podría explicarse por el hecho de que los padres son también parte activa de los cambios en el curso de la vida y la familia, sus propios hijos responden a características que ellos mismos describen en la juventud y ellos mismos son actores activos de las transformaciones que puedan acontecer en la relación de autoridad entre padres-hijos. Desde el rol institucional atribuido $y$ auto-atribuido por los docentes, de formadores tanto académicos como formadores morales $y$ de ciudadanos, la juventud $y$ los padres actuales se alejan del ciudadano ideal (ligado más bien a la reproducción de una sociedad tradicional) e incluso atentan contra la sociedad misma. Desde la perspectiva de los docentes, no solo el joven actúa de manera censurable y riesgosa, los mismos padres con sus omisiones $y$ descuidos participan del actuar juvenil, resultando igualmente censurables.

En cuanto a las estrategias atributivas se recurre a causas externas al explicar la conducta adolescente. Este recurso cognoscitivo no es típico al evaluar el comportamiento de exogrupos, por lo general se recurre a la atribución de rasgos intrínsecos al grupo. Ahora bien, esta estrategia puede ser explicada ya que se considera al joven básicamente incompetente para tomar decisiones por sí-mismo y actuar autónomamente. De hecho los cambios en los roles y la autoridad paterna son concebidos como el principal causante del comportamiento actual. Esta imagen supone una representación familiarista de la sociedad, que nuevamente remite a una sociedad tradicional como imagen prototípica del funcionamiento social ideal. Al fallar la familia, entran en juego otros agentes con una actitud más bien desintegradora de los nexos orgánicos que permitían la reproducción social en el marco del curso de la vida. Dentro de estos agentes, los medios de comunicación son los que cumplen con mayor claridad dicha función, suplantando el rol paterno de autoridad y de transmisor cultural. Los grupos de pares tendrían un fuerte efecto persuasivo, mediatizado por los medios de comunicación. De hecho las relaciones intergeneracionales quedarían trastocadas por este cambio en las funciones de los agentes socializadores.

Las diferencias entre los grupos pertenecientes a las dos situaciones sociales son mínimas y son opacadas por las diferencias entre los grupos institucionales. 


\section{REFERENCIAS BIBLIOGRÁFICAS}

Buchanan, Ch. 'Mothers' "Generalizad Beliefs about Adolescents: Links to Expectations for a specific Child". Journal of Early Adolescence: 23, 2003, 29-50.

Buchanan, Ch. y Holmbeck, G. "Measuring Beliefs about Adolescent Personality and Behavior". Journal of Youth and Adolescence: 27, 1998, 608-627.

Bruner, J. y Tagiuri, R. "The perception of people". En: Lindzey, G. Handbook of Social Psychology. Cambridge. AddisonWesley Publishing, 1956, 634-654.

Büchner, P.; du Bois-Reymond, M.; Ecarius, J. y Krüger, H-H. Teenie-Welten. Opladen: Leske-Budrich. 1998.

Canales, M. y Peinado, A. "Grupos de discusión". En: Delgado, J. y Gutiérrez, J. Método y técnicas cualitativas de investigación en ciencias sociales. Madrid. Síntesis. 1995.

Corsaro, W. The Sociology of Childhood. CA. Pine Forge Press. 1997.

Cubides, H.; Laverde, M. y Valderrama, C. (Eds.) Viviendo a todas. Jóvenes, territorios culturales y nuevas sensibilidades. Bogotá. Siglo del Hombre Editores. 1998.

Elder, G. "Time, Human Agency and Social Change: Perspectives on the Life Course". Social Psychology Quarterly, 57(1), 1994, 4-15.

Epstein, J. Youth Culture. Identity in a postmodern world. London. Sage. 1998.

Erikson, E. Sociedad y adolescencia. Buenos Aires. Paidós. 1972

Farr, R. y Moscovici, S. Social Representations. Cambridge. Cambridge University Press. 1984.
Feixa, C.; Molina, F. y Alsinet, C. Movimientos juveniles en América Latina. Barcelona. Ariel. 2002.

Ferchhoff, W. Jugend an der Wende vom 20. zum 21. Jahrhundert. Lebensformen und Lebensstile. Opladen: Leske + Budrich. 1999.

Fiske, S y Taylor, S. Social Cognition. 2a. Ed. Singapore. McGraw-Hill. 1991.

Flick, U. (Hrsg.). Psychologie des Sozialen. Repräsentationen in Wissen und Sprache. Hamburg. Rowohlts. 1995.

Gaskell, G. "Individual and Group Interviewing". En: Bauer, M. y Gaskell, G. Qualitative Researching with text, imaging and sound. London. Sage. 2000.

Glaser, B. \& Strauss, A. Grounded Theory. Strategien qualitativer Forschung. Göttingen. Huber. 1998.

Hradil, S. Sozialstrukturanalyse in einer fortgeschrittenen Gesellschaft. Leverkusen. Leske \& Budrich. 1987.

Lamnek. Gruppendiskussion. Weinheim. Psychologisches Verlag Union. 1998.

Lobo, I. y Robert, J. La televisión y el niño costarricense. San José. Editorial Universidad de Costa Rica. 1996.

Lüders, C. "Deutungsmusteranalyse. Annäherungen an ein risikoreiches Konzept”. En: Garz, D. \& Kraimer, K. Qualitativ-empirische Sozialforschung. Konzepte, Methoden, Analyse. Opladen. Westdeutscher Verlag. 1991, pp. 377-408.

Lunt, P. $y$ Livingstone, S. "Rethinking the focus groups in media and communication research". Journal of Communication, 1996, 46, 79-98. 
Meeus, W. "Toward a psychosocial analysis of adolescent Identity: An evaluation of the epigenetic theory (Erikson) and the Identity Status Model (Marcia)". En: Meus, W.; de Goede, M.; Kox, W. \& Hurrelmann, K. (Eds.) Adolescence, Careers and Cultures. Berlin: de Gruyter.1992.

Mayring, P.Einführung in die qualitative Sozialforschung. Weinheim. psychologisches Verlagsunion. 1993.

Niessen, M. Gruppen Diskussion. München. Wilhelm Fink. 1979.

Oevermann, U. Zur Analyse der Struktur von sozialen Deutungmustern. Frankfurt. 1973.

Páez, D; Márquez, J. y Insúa, P. "Estructura y procesos de la Cognición Social”. En: Morales, J.; Moya, M.; Rebollosso, E.; et al. Psicología Social. Madrid. Mcgraw Hill. 1998, pp. 123-170.
Peterson, G. \& Peters, D. "Adolescent's Construction of Social Reality. The Impact of Television and Peers". Youth and Society, 15, 1983, 67-85.

Smith, E. \& Queller, S. "Mental representations". En: Tesser, A. y Schawarz, N. Blackwell Handbook of Social Psychology. Intraindividual Processes. Oxford, UK. Blackwell. 2003, pp. 11-133.

Steinke, I. Kriterien Qualitativer Forschung. Weinheim. Juventa. 1999.

Strauss, A. \& Corbin, J. Basics of Qualitative Research. Techniques and Procedures for Developing Grounded Theory. California. Sage. 1998.

Valles, M. Técnicas cualitativas de investigación social. Madrid. Pirámide. 1997.

Wyn, J. y White, R. Rethinking Youth. London, Thousand Oaks \& New Delhi. Sage. 1997. 
